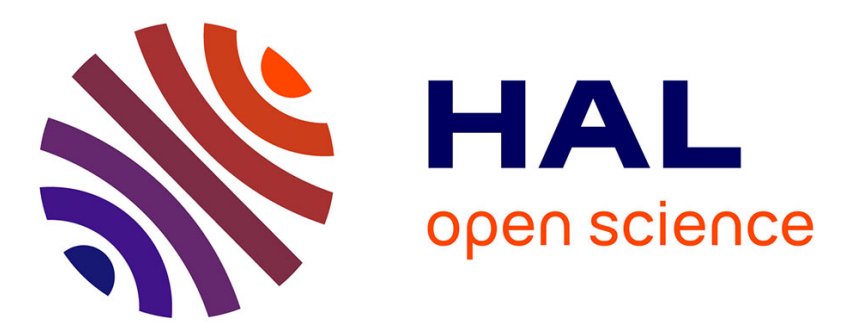

\title{
Etude par photoémission de la passivation de GaAs en plasmas multipolaires d'azote et d'hydrogène
}

\author{
P. Friedel, J.P. Landesman, R. Mabon
}

\section{To cite this version:}

P. Friedel, J.P. Landesman, R. Mabon. Etude par photoémission de la passivation de GaAs en plasmas multipolaires d'azote et d'hydrogène. Revue de Physique Appliquée, 1987, 22 (8), pp.797802. 10.1051/rphysap:01987002208079700 . jpa-00245613

\section{HAL Id: jpa-00245613 https://hal.science/jpa-00245613}

Submitted on 1 Jan 1987

HAL is a multi-disciplinary open access archive for the deposit and dissemination of scientific research documents, whether they are published or not. The documents may come from teaching and research institutions in France or abroad, or from public or private research centers.
L'archive ouverte pluridisciplinaire HAL, est destinée au dépôt et à la diffusion de documents scientifiques de niveau recherche, publiés ou non, émanant des établissements d'enseignement et de recherche français ou étrangers, des laboratoires publics ou privés. 


\title{
Etude par photoémission de la passivation de GaAs en plasmas multipolaires d'azote et d'hydrogène
}

\author{
P. Friedel, J. P. Landesman et R. Mabon \\ Laboratoires d'Electronique et de Physique appliquée (LEP) (*) 3 av. Descartes, 94450 Limeil-Brevannes, \\ France
}

(Reçu le 16 janvier 1987, révisé le 27 avril 1987, accepté le 28 avril 1987)

\begin{abstract}
Résumé. - La passivation des matériaux III-V demeure un défi aux physiciens des surfaces. Un processus de passivation faisant intervenir une étape de nettoyage et la formation d'un nitrure en plasma a été proposé. Dans cette revue des résultats obtenus au LEP, nous détaillerons particulièrement l'interaction entre le plasma d'hydrogène ou le plasma d'azote et la surface de l'échantillon. Le plasma multipolaire utilisé produit des ions peu énergétiques et une forte proportion d'espèces atomiques. Nous concentrons notre investigation à l'étude des liaisons chimiques observées par photoémission et aux propriétés électriques de la surface. Les caractéristiques électriques sont étudiées in situ par photoémission de niveaux de cœur. L'attention sera focalisée sur la présence d'arsenic "élémentaire » et sur la désoxydation de la surface.
\end{abstract}

\begin{abstract}
The passivation of III-V materials is a challenge to surface physicists. A passivation process by plasma cleaning and nitriding the surface has been proposed. This review of the results obtained at LEP on this subject mainly concentrates on the interaction between the hydrogen plasma or the nitrogen plasma and the surface of the sample. The multipolar plasma generates low temperature ions and a high proportion of atomic species. We are concerned with the chemical bonds as revealed by photoemission and by the electrical properties of the surface. The electrical properties are measured in situ by core level photoemission. We shall center our investigations on the existence of « elemental» arsenic and on the oxide removal on the surface.
\end{abstract}

De façon à réduire les effets parasites sur les transistors MESFET nous recherchons un traitement en plasma qui produise une bonne interface isolantsemiconducteur. Le plasma que nous avons choisi est une décharge à cathode chaude associée à un confinement magnétique (plasma multipolaire). Une séquence de désoxydation suivie d'une nitruration native en plasma et d'un dépôt de nitrure de silicium a été proposée [1]. Elle a conduit à des réductions substantielles de courants de fuite entre la source et la grille ainsi qu'une diminution de la dispersion en fréquence de la transconductance. Le même traitement conduit sur $\mathrm{Ga}_{0,47} \mathrm{In}_{0,53}$ As à une densité d'états de surface inférieure à $3 \times$ $10^{11} \mathrm{~cm}^{-2} \mathrm{eV}^{-1}$. Dans cet article, nous analysons des surfaces de GaAs (100) de type $n$ et de type p par photoémission (XPS : X-ray photoemission spectroscopy). La surface est étudiée avant et après l'exposi-

(*) LEP, Laboratoires d'Electronique et de Physique appliquée : un membre de l'organisation internationale de recherche Philips. tion à un plasma d'hydrogène ou d'azote. Le traitement et l'analyse sont effectués dans un système entièrement sous ultra-vide.

\section{Le montage expérimental.}

Le spectromètre est un analyseur hémisphérique Leybold EA11 ; le rayonnement X provient soit de la raie $K \alpha$ de l'aluminium, soit de la raie $K \alpha$ du magnésium. L'énergie du photon est alors respectivement soit $1486,7 \mathrm{eV}$ soit $1253,6 \mathrm{eV}$. Un système de transfert permet de déplacer l'échantillon de la chambre d'analyse à la chambre à plasma. La pression de l'enceinte est inférieure à $10^{-9}$ torr durant les transferts. Elle atteint $10^{-10}$ torr dans la chambre d'analyse.

Les échantillons sont des plaques de GaAs découpées dans le plan (100). Ils subissent un polissage mécanochimique. Les échantillons de type $n$ sont dopés par le sélénium à $4 \times 10^{17} \mathrm{~cm}^{-3}$, tandis que les échantillons de type $\mathrm{p}$ sont dopés par le cadmium de 3 à $5 \times 10^{18} \mathrm{~cm}^{-3}$. 
Les spectres de photoémission sont déconvolués en utilisant une méthode originale des moindres carrés. L'originalité réside dans le choix du spectre représentatif des composantes élémentaires. Les méthodes de déconvolution par moindres carrés utilisées habituellement font appel à la reconstruction d'un spectre «théorique " par élargissement de pics de Dirac par des gaussiennes et des lorentziennes. Ces fonctions peuvent éventuellement être rendues asymétriques pour rendre compte d'effets à plusieurs électrons. Dans le cas d'un spectre non monochromatisé comme ceux que nous mesurons, il faudrait inclure la forme exacte de la raie d'émission $X$ avec le détail des quatre satellites $K \alpha_{1}$, $\mathbf{K} \alpha_{2}, \mathbf{K} \alpha_{3}$ et $\mathbf{K} \alpha_{4}$. Ceci demande une connaissance très précise des caractéristiques de la source de rayons $\mathrm{X}$. Généralement, le recours à de telles techniques conduit à des résultats de déconvolution qui dépendent des choix initiaux des paramètres et des formes des fonctions d'élargissement. Nous avons cherché à minimiser ce problème en ayant recours à des spectres « idéaux » mesurés expérimentalement sur des surfaces propres du matériau semiconducteur qui nous intéresse. Les spectres de base que nous déterminons ainsi sont pris dans des conditions de sensibilité maximum au volume du matériau. On évite ainsi de faire intervenir dans le spectre utilisé pour les déconvolutions des contributions de composés présents en surface du matériau. Pour déterminer si une surface est effectivement propre, nous avons comparé un spectre mesuré en émission normale (sensibilité au volume) et en émission rasante (sensibilité à la surface). Nous avons retenu pour le choix des pics utilisés pour les déconvolutions, des échantillons pour lesquels les pics de volume et de surface sont superposables. Le résultat des déconvolutions est alors très peu sensible aux conditions initiales du processus d'approche aux moindres carrés.

La sensibilité à la surface est intéressante pour étudier les composés présents initialement ou formés par les interactions avec le plasma. Elle peut être réalisée en inclinant l'échantillon de façon à ce que les électrons analysés soient émis dans une direction rasante. Elle peut aussi être réalisée en choisissant un des pics d'énergie cinétique plus faible. Il est donc possible de confirmer tous nos résultats en utilisant les niveaux $2 p$ de l'arsenic et du gallium. La sensibilité à la surface pour ce pic est alors équivalente à celle que l'on obtient avec le pic $3 \mathrm{~d}$ à $75^{\circ}$ d'angle d'émission. Le libre parcours moyen inélastique effectif le long de la normale à la surface est alors de $7 \AA$.

\section{Caractérisation de la surface initiale.}

Les échantillons subissent un traitement de désoxydation par voie chimique juste avant leur introduction dans l'enceinte :
- dégraissage dans une solution bouillante éthanol-trichloréthylène-acétone,

- désoxydation dans une solution à $1: 10$ d'acide chlorhydrique et d'éthanol,

- rinçage dans l'éthanol bouillant.

La solution $\mathrm{HCl} / \mathrm{C}_{2} \mathrm{H}_{5} \mathrm{OH}$ conduit à une contamination minimum en carbone tout en maintenant la stœechiométrie de surface du $\mathrm{GaAs}^{2}$ [2].

Nous montrons sur la figure $1 \mathrm{~b}$ les spectres $3 \mathrm{~d}$ de l'arsenic et du gallium mesurés en émission rasante. La déconvolution des spectres d'arsenic révèle la présence d'une contribution décalée de $0,6 \pm 0,1 \mathrm{eV}$ vers les plus hautes énergies de liaison. Cette valeur est en accord avec les valeurs publiées pour l'arsenic élémentaire. Un excès d'arsenic est donc en général observé sur la surface nettoyée chimiquement. Les deux spectres de la figure 1 sont révélateurs d'un reliquat d'oxyde. Nous montrerons plus loin comment l'épaisseur d'oxyde a pu être estimée. Les décalages en énergie indiquent que l'oxyde correspond en grande partie à la stœchiométrie $\mathrm{As}_{2} \mathrm{O}_{3}$ et $\mathrm{Ga}_{2} \mathrm{O}_{3}$. Il est fort possible que d'autres formes oxydées soient aussi présentes sur cette surface.

\section{SENSIBILITE VOLUME - SURFACE}

VOLUME : EMISSION NORMALE FORTES ENERGIES CINETIQUES
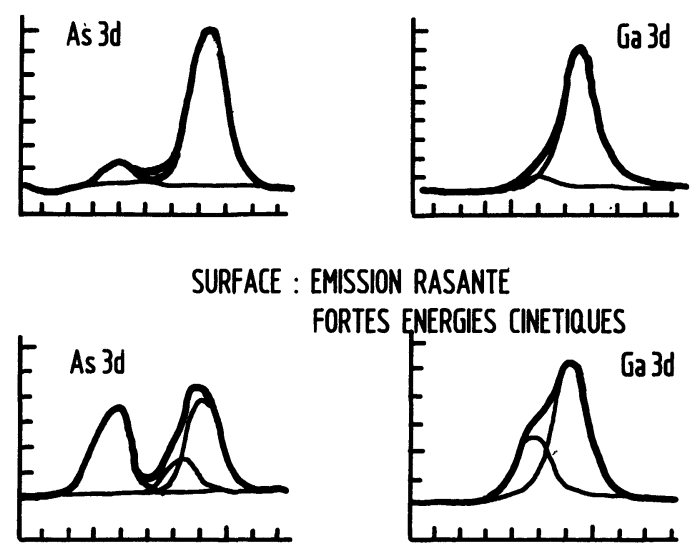

SURFACE : EMISSION NORMALE

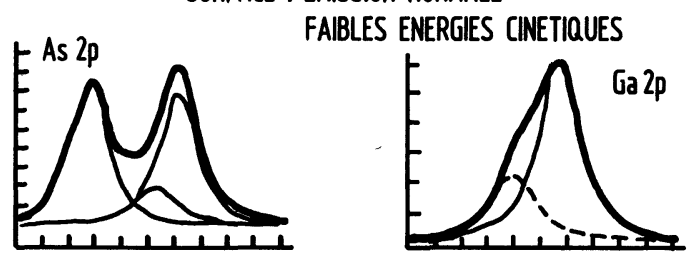

Fig. 1. - Spectres de l'arsenic et du gallium en surface. Les spectres mesurés en émission rasante et à basse énergie cinétique indiquent la présence d'oxydes et d'arsenic élémentaire sur la surface initiale.

[Surface spectra of arsenic and gallium after chemical cleaning. The spectra in grazing emission and those at low kinetic energy are indicative of oxides and elemental arsenic on the chemically cleaned surface.] 
Du carbone est toujours présent sur la surface (il est révélé par la présence du pic $1 \mathrm{~s}$ du carbone que nous présentons par ici).

\section{Le nettoyage en plasma d'hydrogène.}

Nous avons optimisé les paramètres du plasma de façon à nettoyer l'échantillon en maintenant la stœchiométrie de surface. Pour une pression de l'ordre de $4 \times 10^{-4}$ torr, un courant de décharge de $100 \mathrm{~mA}$, une tension de décharge de $-75 \mathrm{~V}$ et une température de l'échantillon de l'ordre de $200{ }^{\circ} \mathrm{C}$, la densité électronique est dans la gamme $10^{10} \mathrm{~cm}^{-3}$.

Dans ces conditions, le retrait de l'oxyde et du carbone est total à la fois sur les échantillons de type $n$ et de type $p$ après une minute de plasma (Fig. 2). Il est nécessaire de chauffer l'échantillon pour éliminer les oxydes de gallium. A basse température [3], l'oxyde de gallium est réduit de la forme $\mathrm{Ga}_{2} \mathrm{O}_{3}$ à une forme où le degré d'oxydation du gallium est de l'ordre de 1 . Nous pouvons penser à la forme peu oxydée $\mathrm{Ga}_{2} \mathrm{O}$. Le pic 1 s de l'oxygène disparaît totalement juste après le traitement à chaud; il peut réapparaître après $10 \mathrm{~h}$ de stockage sous ultra-vide (à $10^{-10}$ torr). En fait, l'oxygène est alors seulement physisorbé sur la surface; il peut être évaporé de la surface par un simple chauffage avant tout traitement ultérieur.

On peut profiter du fait que la surface est nettoyée de ses contaminants pour évaluer l'épaisseur d'oxyde initialement présente sur la surface. Nous utilisons pour cela un modèle à une couche fine homogène sur la surface. Nous assimilons le libre parcours moyen inélastique des électrons à la profondeur d'échappement. L'épaisseur d'oxyde est alors reliée au rapport d'intensité du pic de volume d'un atome donné sur la surface initiale, $I^{\prime}$, et sur la surface propre, $I$, par l'expression :

$$
E_{\mathrm{OX}}=-\lambda \cos \phi \log \frac{I^{\prime}}{I}
$$

TRAITEMENTS DE DESOXYDATION EMISSION RASANTE

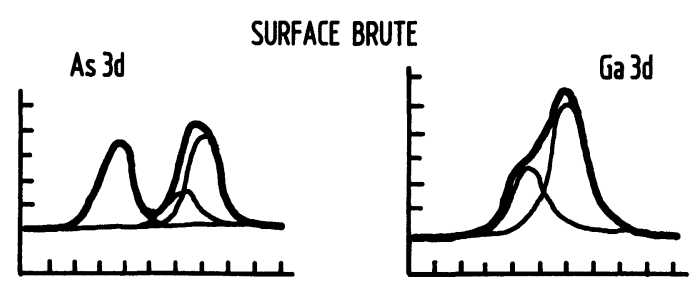

(a)

\section{CHAUFFAGE A $290^{\circ} \mathrm{C}$}
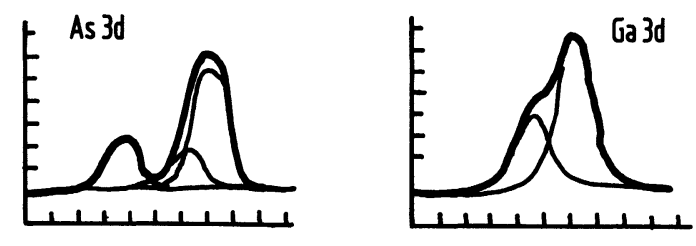

PLASMA A BASE D'HYDROGENE
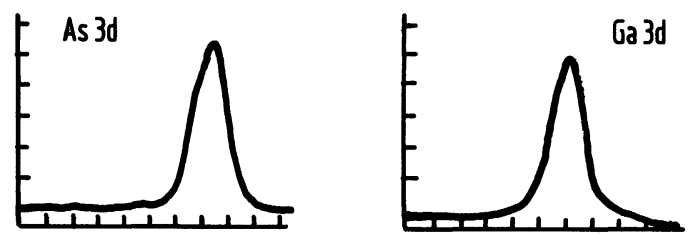

(c)

Fig. 2. - Spectres 3d de As et Ga en surface durant le traitement plasma. L'oxyde et l'arsenic élémentaire sont réduits dans un plasma d'hydrogène.

[Surface 3d spectra of As and Ga during the plasma treatment. The oxide and the elemental arsenic are reduced in a hydrogen plasma.]

où $\phi$ est l'angle d'émission des électrons. On obtient ainsi sur l'ensemble des échantillons que nous avons étudiés, les résultats reportés dans le tableau I.

L'épaisseur d'oxyde a été évaluée à partir de l'intensité des niveaux $3 \mathrm{~d}$ de l'arsenic et du gallium en émission normale êt en émission rasante ainsi que

Tableau I. - Epaisseurs d'oxyde mesurées par photoémission à partir de différents niveaux de coeur. La première colonne correspond à des niveaux $3 \mathrm{~d}$ mesurés en émission normale; la seconde en émission rasante; enfin la troisième colonne a été obtenue à partir de la composante de spin total $3 / 2$ des niveaux $2 \mathrm{p}$. A chaque fois le gallium et l'arsenic ont été utilisés.

[Oxide thicknesses measured by photoemission using different core levels. The first column corresponds to $3 \mathrm{~d}$ levels measured in normal emission. The second is in grazing emission. The third has been obtained using the $3 / 2$ total spin component of the $2 p$ levels. In each instance, the evaluation was made both for gallium and for arsenic.]

\begin{tabular}{|l|c|c|c|c|c|c|}
\hline & \multicolumn{2}{|c|}{$3 \mathrm{~d}_{\perp}$} & \multicolumn{2}{c|}{$3 \mathrm{~d}_{\|}$} & \multicolumn{2}{c|}{$2 \mathrm{p}^{3 / 2}$} \\
\hline & $\mathrm{As}$ & $\mathrm{Ga}$ & As & $\mathrm{Ga}$ & As & $\mathrm{Ga}$ \\
\hline Surface initiale & $3 \rightarrow 9 \AA$ & $4 \rightarrow 13 \AA$ & $2 \rightarrow 8 \AA$ & $5 \rightarrow 7 \AA$ & $\sim 7 \AA$ & $\sim 6 \AA$ \\
\hline Après chauffage & $1 \rightarrow 5 \AA$ & $1 \rightarrow 6 \AA$ & $2 \rightarrow 7 \AA$ & $2 \rightarrow 6 \AA$ & $2 \rightarrow 7 \AA$ & $2 \rightarrow 7 \AA$ \\
\hline
\end{tabular}


du niveau $2 p 3 / 2$ en émission normale. La bonne correspondance entre les résultats obtenus est une indication que l'ordre de grandeur trouvé est correct. Nous avons évalué l'incertitude sur la valeur trouvée à environ $60 \%$. Ces échantillons ont été observés par ellipsométrie par P. Boher. Les épaisseurs d'oxyde qu'il détermine sont systématiquement plus fortes d'un facteur de l'ordre de 2. Une étude assez détaillée de cette différence a été menée en prenant comme mesure de référence des sections transverses en microscopie à haute résolution [4]. On peut attribuer la différence à la présence d'une interface rugueuse ; elle est non visible par photoémission; elle contribue largement à la mesure en ellipsométrie.

Les déplacements de niveaux de cour de l'arsenic ou du gallium par liaison avec le carbone ne sont pas répertoriés dans la littérature à notre connaissance. Nous avons suivi le retrait de carbone lors d'expositions à des plasmas à basse température de durée croissante. Les niveaux de l'arsenic, du gallium et du carbone ont été relevés pour la séquence de plasmas suivante :

1. surface initiale,

2. plasma de $5 \mathrm{~s}$,

3. nouveau plasma de $5 \mathrm{~s}$ sur le même échantillon (exposition totale $10 \mathrm{~s}$ ),

$20 \mathrm{~s})$

5. etc... jusqu'à une exposition totale de $160 \mathrm{~s}$.

Le fractionnement et l'addition de durées de plasma sont équivalents à des plasmas continus de durée égale au total comme en témoignent les mesures faites sur un autre échantillon avec les durées de $80 \mathrm{~s}$ et $160 \mathrm{~s}$ de plasma continu. Le résultat de cette séquence est représenté sur la figure 3 . On

\section{SEQUENCE DE 8 PLASMAS D'HYDROGENE}

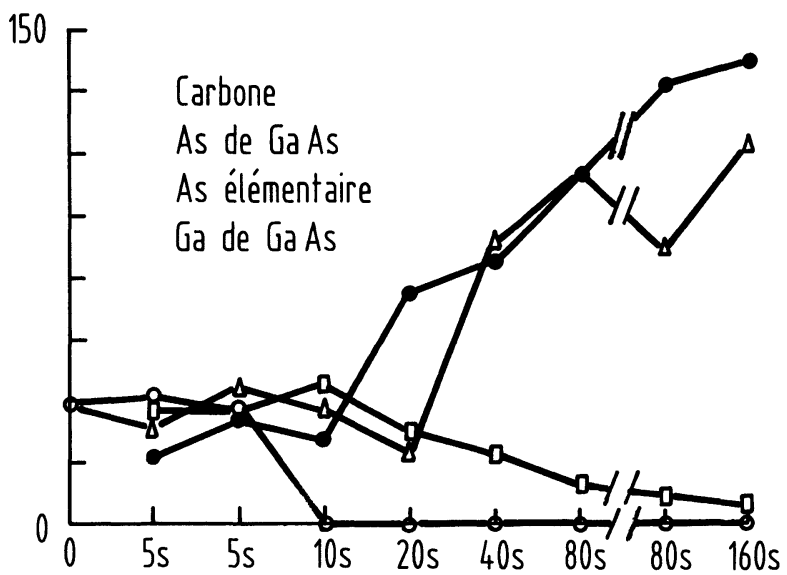

Fig. 3. - Cinétique d'une exposition au plasma d'hydrogène à froid.

[Kinetics of the exposure to a hydrogen plasma at room temperature.] constate que l'arsenic élémentaire disparaît très tôt et que le carbone est réduit pour des expositions plus longues. Parallèlement à la disparition du carbone, les contributions de volume de l'arsenic et du gallium augmentent dans des proportions comparables. Ceci peut être dû à un effet de profondeur d'échappement des électrons. Cela pourrait être expliqué par l'existence d'une couche fine de carbone ou d'hydrocarbure sur la surface initiale ; le plasma d'hydrogène fait disparaître cette couche. L'examen plus approfondi du pic $1 \mathrm{~s}$ du carbone devrait révéler la présence de liaisons avec l'hydrogène [5]. La présence d'un pic Auger de l'arsenic à proximité immédiate du niveau 1s du carbone rend toute interprétation douteuse.

Les conditions de plasma citées plus haut sont optimales du point de vue de la stœechiométrie de surface. L'excès d'arsenic élémentaire disparaît et le rapport du gallium de volume et de l'arsenic de volume est très voisin de 1 . Nous avons étudié l'effet du plasma d'hydrogène sur la stœchiométrie des surfaces obtenues par épitaxie aux jets moléculaires $[6,7]$. On a pu voir que l'hydrogène interagit en deux temps sur la surface stabilisée en arsenic et reconstruite $2 \times 4$. Pour des plasmas de faible intensité et de courte durée, l'hydrogène s'attache à l'arsenic de surface. Une étude détaillée des bandes de valence permet de penser que l'arsenic se lie aussi à du gallium dans cette première phase. Pour des plasmas plus longs, la composante de niveau de cœur correspondant à l'arsenic hydrogéné disparaît ; l'hydrogène a probablement formé des hydrures d'arsenic volatils, sans doute $\mathrm{AsH}_{3}$. Dans cette phase, on constate effectivement une diminution sensible du rapport d'intensité du pic $3 \mathrm{~d}$ d'arsenic et du pic de gallium.

On peut alors se demander à quel point le plasma d'hydrogène permettrait de graver la surface. Pour répondre à cette question, nous avons effectué un plasma d'hydrogène à froid, dans les conditions par ailleurs optimales. Un échantillon partiellement recouvert de laque ultra-vide a été exposé au plasma pendant une heure. Contrairement à ce qui a été observé sur d'autres types de plasmas [8], nous ne constatons alors aucune différence de niveau entre la surface sous la laque et la surface soumise au plasma. Nous pouvons affirmer que si le plasma grave la surface, l'épaisseur gravée n'excède pas $50 \AA \AA$. On doit donc s'attendre à une gravure inférieure à un ou deux plans atomiques pendant une minute de plasma de nettoyage. Il semble donc que le plasma multipolaire d'hydrogène grave préférentiellement les plans d'arsenic et que la gravure soit bloquée par les plans de gallium. Nous n'avons jamais constaté de disparition massive d'arsenic au point de laisser sur la surface des gouttelettes de gallium, comme nous aurions pu nous y attendre.

Un point important est fourni par les mouvements 
d'ensemble des niveaux de cœur du gallium et de l'arsenic. Les uns comme les autres sont décalés après le plasma par rapport à leur position précédant le plasma. Du point de vue expérimental, on peut se demander si ce décalage est un artefact de la mesure ou bien correspond à un effet électronique dans l'échantillon. Il est possible que le plasma d'hydrogène modifie la conductivité de l'échantillon. Il agirait alors sur l'équilibre dynamique qui régit la neutralité de l'échantillon sous le faisceau de rayons $X$. La charge positive créée par l'émission de photoélectrons est contrebalancée :

- par le courant de conduction provenant de la face arrière de l'échantillon,

- et par le courant d'électrons de basse énergie émis par le rayonnement de freinage au passage de la fenêtre en aluminium qui isole la source de l'échantillon.

Pour un échantillon bon conducteur, le courant de conduction est suffisant pour assurer la neutralité. Si l'échantillon devient isolant, le courant de face avant prend de l'importance. Pour tester ce point, il suffit de changer la puissance de la source $X$. Nous avons mesuré les déplacements des niveaux de cœur en fonction de la puissance de la source $X$. Aucune corrélation n'a été constatée entre ces deux grandeurs ; le décalage a donc une autre origine qu'un changement de conductivité de l'échantillon. Rappelons que la référence des mesures de photoémission dans un solide est le niveau de Fermi, corrigé par le travail de sortie de l'analyseur. Le travail de sortie de l'échantillon n'intervient que par un effet de seuil vers les basses énergies cinétiques. Les mouvements d'ensemble que nous observons sont donc liés à des déplacements du niveau de Fermi dans la bande interdite. Sur type $\mathbf{n}$ comme sur type $\mathrm{p}$, le même décalage est observé ; il baisse le niveau de Fermi de $200 \pm 50 \mathrm{meV}$ vers le maximum de la bande de valence. Il correspond donc à une diminution de la courbure de bandes sur type p et à une augmentation sur type n. On peut se demander si ceci est un effet de volume ou un effet de surface seulement. Une adsorption d'oxygène moléculaire à température ambiante ramène le niveau de Fermi à sa position initiale. Il est donc très probable que l'effet observé soit exclusivement un effet de surface.

\section{Nitruration plasma.}

L'exposition au plasma d'azote a été effectuée sur les deux types de dopage. La pression d'azote dans le plasma est maintenue à $4 \times 10^{-3}$ torr, la tension de décharge est de $-75 \mathrm{~V}$ et le courant de décharge de $100 \mathrm{~mA}$. L'échantillon est chauffé à $230^{\circ} \mathrm{C}$ pendant une minute avant le démarrage de la décharge. On évapore ainsi l'oxygène qui s'est adsorbé sur la surface propre pendant les mesures de photoémis-
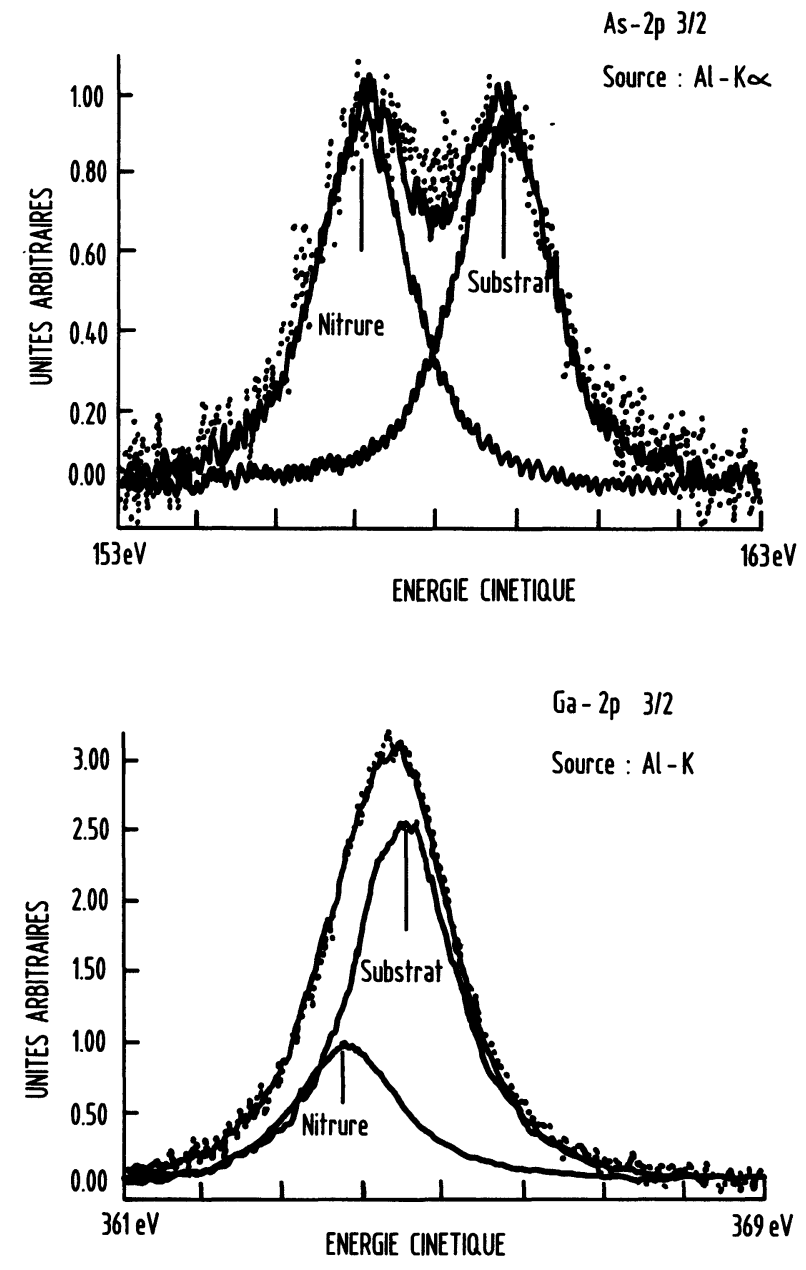

Fig. 4. - Spectres As et Ga 2p3/2 après le plasma d'azote.

[As and $\mathrm{Ga} 2 \mathrm{p} 3 / 2$ spectra after the nitrogen plasma.]

sion. On évite ainsi de greffer l'oxygène à la surface pendant le plasma.

La figure 4 fait apparaître les composantes du nitrure sur les niveaux du gallium et de l'arsenic. On a pu vérifier que ces composantes existent en absence d'oxygène sur la surface. Elles correspondent bien à un nitrure. Toutefois, selon les conditions de plasma et surtout de préchauffage de la surface juste avant le plasma d'azote, on peut observer de l'oxygène sur la surface. Ces résultats sont une confirmation de ceux qui avaient été obtenus sur la surface épitaxiée aux jets moléculaires [9]. Sur type $p$, un mouvement d'ensemble des niveaux de cœur est observé. Nous l'avons traduit sur la figure 5 par un mouvement du niveau de Fermi. La nitruration conduit à des positions de niveau de Fermi variables. Elles dépendent peut-être de l'état d'oxydation de la surface. Sur type $n$, les niveaux sensibles à la surface - niveaux $3 \mathrm{~d}$ en émission rasante et niveaux $2 \mathrm{p}$ - accusent un décalage plus important (de l'ordre de $500 \mathrm{meV}$ ). Il est possible qu'un dipôle soit engendré sur quelques plans atomiques près de la surface nitrurée sur type $n$. 
MOUVEMENTS DU NIVEAU DE FERMI

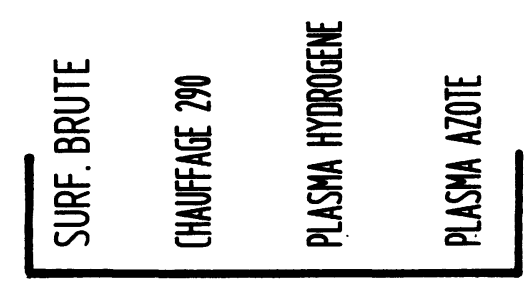

$1,43 \mathrm{eV}$

type $n$

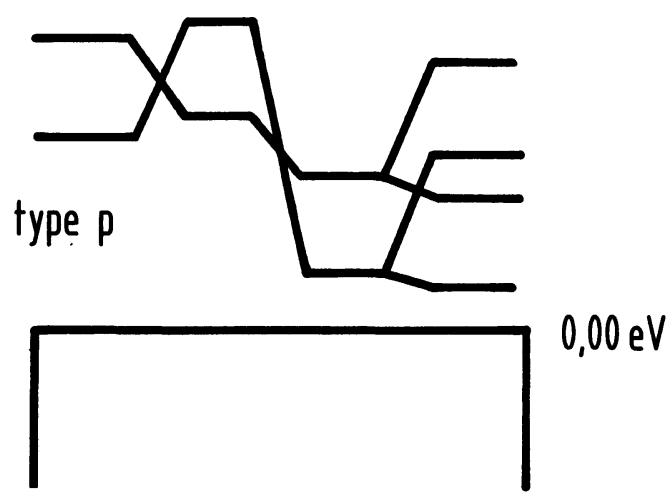

Fig. 5. - Mouvements du niveau de Fermi durant l'ensemble du traitement.

[Fermi level movements during the whole treatment.]
Sur le type $\mathrm{p}$ les mesures de photoémission sont confirmées par des mesures d'ellipsométrie spectro scopique [10]; la double structure à la transition $\mathrm{E}_{1}, \mathrm{E}_{1}+\Delta$ est sensible au champ électrique dans la zone de courbure de bandes. L'évolution de cette structure reproduit les mouvements observés du niveau de Fermi en photoémission. Par contre, sur type $n$, la double structure est moins sensible au champ électrique et d'autres effets sont peut-être à l'origine de la différence de résultats entre ellipsométrie et photoémission. Il reste à éclaircir l'influence éventuelle d'un dipôle induit par l'azote sur cette surface.

\section{Conclusion.}

L'étude fine du nettoyage en plasma d'hydrogène et de la nitruration en plasma d'azote a clairement mis en évidence un déplacement du niveau de Fermi sur les surfaces de GaAs au moins sur le type p. L'étude des niveaux de cœur des atomes du substrat ainsi que des contaminants a montré la possibilité de réduire l'oxygène, le carbone et l'arsenic élémentaire en plasma d'hydrogène. Le plasma d'azote crée une interface abrupte [11] et conduit à plusieurs positions du niveau de Fermi dans la bande interdite. Ceci est une indication que la position de blocage habituelle a été modifiée et peut expliquer l'amélioration des propriétés électroniques des dispositifs passivés de cette façon [1].

\section{Bibliographie}

[1] Demay, Y., Thèse de $3^{\mathrm{e}}$ cycle, Paris 1982.

[2] Vasquez, R. P., Lewis, B. F., Grunthaner, F. J., J. Vac. Sci. Technol. B 1 (1983) 791.

[3] Gourrier, S., Friedel, P. et Larsen, P. K., Surf. Sci. 152/153 (1985) 1147.

[4] Mabon, R., Boher, P., Barrois, A., Hily, Y., LANDESMAN, J. P. et Friedel, P., à paraître.

[5] SiegbAhN, K. et al., ESCA, atomic, molecular and solid state structure studied by means of electron spectroscopy. Nova Acta Regiae Soc. Sci. Ups. IV (20) (1967).

[6] Friedel, P., Larsen, P. K., Gourrier, S., CabaNIE, J. P. et Gerits, W. M., J. Vac. Sci. Technol. B 2(4) (1984) 675.
[7] Carette, T., lannoo, M., Allan, G., Friedel, P., Surf. Sci. 164 (1985) 260.

[8] Chang, R. P. H. et Darack, R. S., App. Phys. Lett. 38 (1981) 898.

[9] Gourrier, S., Smit, L., Friedel, P. et Larsen, P. K., J. Appl. Phys. 54 (1983) 3993.

[10] Landesman, J. P., Friedel, P., Boher, P., SchneIDER, J., Proceedings of the 14th PCSI conference (1987), à paraître dans J. Vac. Sci. Technol. (1987).

[11] Ruterana, P., Friedel, P., Schneider, J. et Chevallier, J. P., Appl. Phys. Lett. 49 (1986) 672. 